\title{
LASER RANGING MODELING UNDER GENERALIZED MIXED PIXELS EFFECT
}

\author{
C. M. Chang ${ }^{1}$, J. J. Jaw ${ }^{1 *}$ \\ ${ }^{1}$ Dept. of Civil Engineering, National Taiwan University, Taipei, Taiwan - (r07521805, jejaw)@ntu.edu.tw
}

KEY WORDS: Footprint, Generalized mixed pixels effect, Incidence angle, Laser ranging, Systematic error

\begin{abstract}
:
When a target lies on discontinuous surfaces, the footprint of a laser rangefinder covering multiple ranges causes mixed pixels effect and significantly distorts the ranging quality. Meanwhile, the ranging error of incidence angle effect is triggered by a deformed footprint containing various ranges as well. Based on the commonality of causing ranging errors within one footprint, this study proposed an approach to tackle "generalized mixed pixels effect" correcting ranging errors involving in deformed footprint cases. Errors caused by generalized mixed pixels effect vary in rangefinders and are difficult to be uniformly treated. A correction model was formulated through integrating individual effects by considering the physical and geometrical aspects of laser ranging. An adjustment procedure was followed to estimate the parameters of the correction equation taking all observation uncertainties into account. To analyze the individual effects and eventually combine them into a complete model, a five-case workflow has been developed. Firstly, a divergence angle estimation method was presented to eliminate the mixed pixels effect by a decentering approach. Incidence angle effect was modeled and parameter was estimated by adjustment techniques. Particularly, since incidence angles are usually unknown in field surveys, an iterative estimation procedure was designed to obtain the optimal incidence angle of target points. Finally, offset correction accounting for generalized mixed pixels effect was formulated. Through the experimental tests on Trimble M3 DR 2" and Topcon GPT-3002LN, it is confirmed that the proposed method effectively resolves the ranging errors and preserves the ranging quality under generalized mixed pixels effect.
\end{abstract}

\section{INTRODUCTION}

A pulsed time-of-flight laser ranging technique with centering and horizontalization has been widely applied to acquire highquality ranges of interest. Through pulse timing estimators (Abshire et al., 1994), timing from returning pulses is to be determined, and fine ranging data can be obtained in most cases (Adams, 1993). However, generalized mixed pixels effect, including mixed pixels effect and incidence angle effect, breaks the balance.

Mixed pixels effect leads to a serious systematic ranging error because a transmitted laser beam splits and falls on targets with depth (Herbert, Krotkov, 1992). To preclude the ranging error, some instruments are designed to identify the reflection from the target point and filter out the superfluous signals measured from surrounding surfaces (Typiak, 2008). However, as long as a split-up footprint is shorter than the range resolution, the shortest distance a ranging instrument can distinguish between two target points, mixed pixels effect will still exist and distort ranging quality. Range resolution can be calculated by speed of light and a pulse width given in instruction manuals. As shown in Fig. 1(a), with only one laser pulse return, mixed pixels effect deceives the rangefinder with the footprint just involving one target. Thus, unreliable ranging data is responded by the distorted laser pulsed wave (Xiang, Zhang, 2001). On the other hand, if the range resolution is shorter than the depth, the returning pulse wave with multiple crests clarifies individual timing for the rangefinder to compute the corresponding ranging data (Fig. 1(b)). The characteristic of one footprint contaminated by multiple ranges can also be found in incidence angle effect. Geometrically, an emitted footprint will be elongated and deformed because of incidence angles and makes a footprint respond numerous range information. Based on Lambertian scattering law, the incidence angle effect, with a weaker reflected signal in magnitude and wider in time, leads to ranging errors (Soudarissanane et al., 2009). As shown in Fig. 2, fieldwork surveys often involve in points of interest with generalized mixed pixels effect, which can cause significant systematic ranging errors. Therefore, a reliable and effective correction workflow for restoring the ranging quality under generalized mixed pixels effect must be developed.

Corrections of generalized mixed pixels effect are different among rangefinders since signal processing algorithms are varied. It is difficult, if not impossible, to assess the ranging quality in a general form. Analyzing the waveform of a return pulse in time-intensity (or time-amplitude) observations is crucial to range measurement. As the intensity is converted from the received laser pulse power, once a received laser power changes, the ranging results will change as well. Thus, numerous studies have clarified the entangled ranging errors by analyzing intensity information (Kaasalainen et al., 2011; Tan et al, 2018). However, most of the rangefinders do not reveal the waveform information for general users to explore the intensityrange relationship (Adams, Probert, 1996). This means that even though intensity is the key to estimating the generalized mixed pixels effect, the intensity data should be seen as unknown.

The aim of this study is to develop a workflow fulfilling the fieldwork ranging quality under generalized mixed pixels effect (Fig. 2) without waveform data. The proposed workflow modeled and derived the parameters of correction equations purely based on an initiative realization from the physical property and range observations. The proposed five-case workflow can be adapted to most laser rangefinders and ultimately eliminates or reduces the ranging errors under generalized mixed pixels effect. This paper is structured in four

* Corresponding author 
sections. The details of the proposed workflow are introduced in Section 2. In Section 3, experiment results of the developed procedure applied on two total stations with the pulse time-offlight laser ranging technique are provided. Conclusion in Section 4 is drawn.
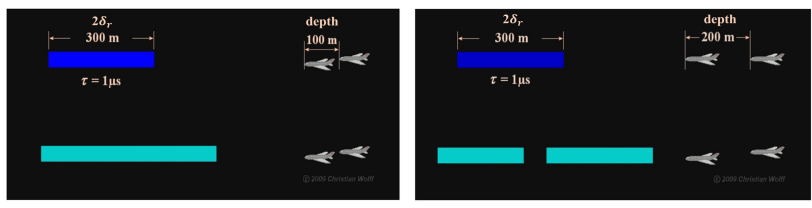

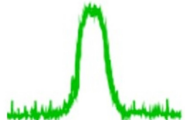

(a)

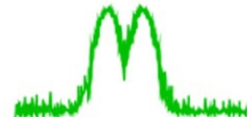

(b)
Figure 1. Illustration of range resolution (modified from Wolff, 2009). (a) the depth is shorter than the range resolution $\left(\delta_{r}\right)$ rendering a deformed one-crest laser pulse; (b) the depth is longer than the range resolution and a deformed pulse return with two wave crests is obtained

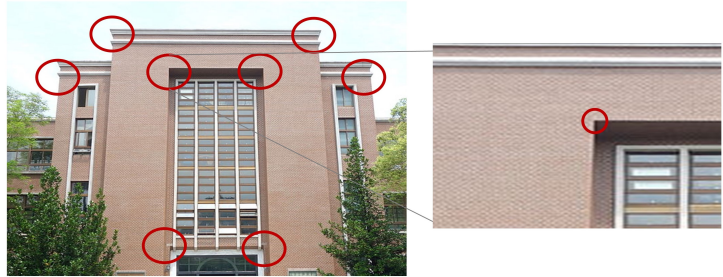

Figure 2. Targets with generalized mixed pixels effect

\section{LASER RANGING MODELING WORKFLOW}

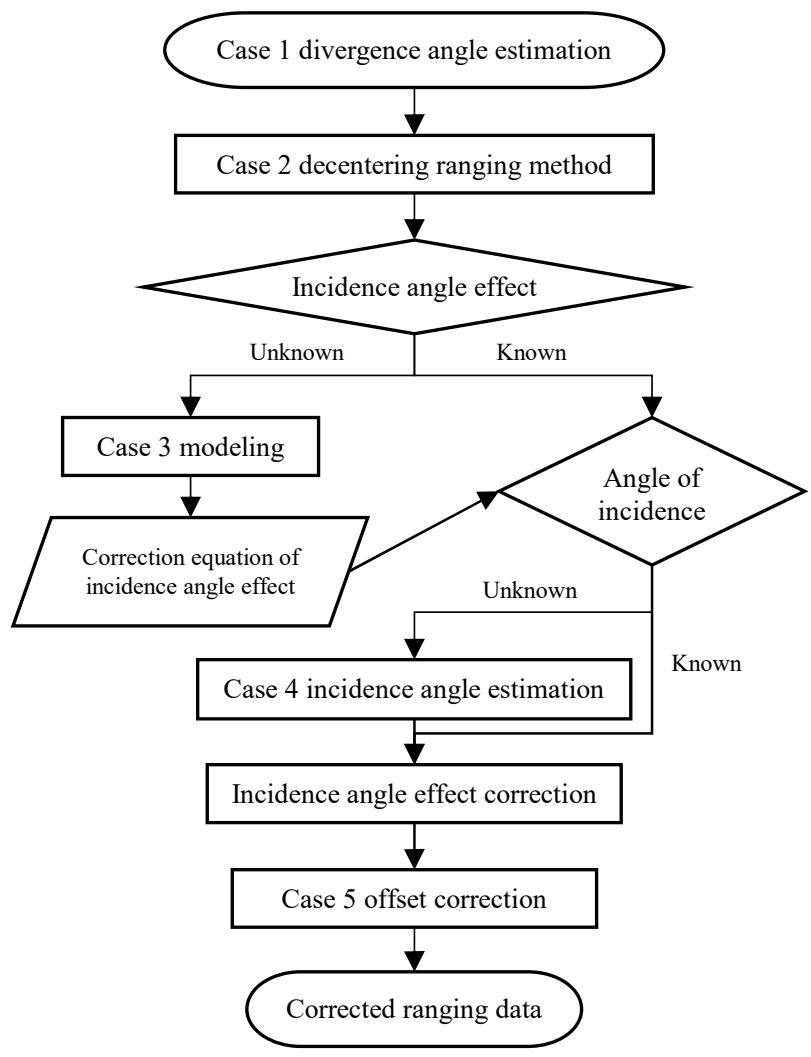

Figure 3. The proposed workflow chart
Analyzing the geometric components of laser rangefinder measurement, the main factors of generalized mixed pixels effect may include the divergence angles of laser rangefinders, incidence angles, and distances. By decomposing the generalized mixed pixels effect based on the geometric consideration, a five-case workflow and the related algorithms have been developed in this study to dissolve the laser ranging errors under generalized mixed pixels effect. The scheme of the proposed workflow is illustrated in Fig. 3.

\subsection{Case 1: divergence angle estimation}

Mixed pixels effect occurs when a footprint contains multiple ranges; therefore, before modeling this effect, the laser footprint dimension of the applied instrument is required. Even if the laser beam, the red area in Fig. 4(a), covers not only the round foreground but also the background, the ranging results do not necessarily involve in mixed pixels effect. It can be stated that the red projected area of a laser beam does not represent the emitted footprint. To further verify a footprint diameter, one should refer to an instruction manual or an estimation approach detailed as follows. As shown in Fig. 4(b), it is assumed that the footprint is a round shape and the radius of it awaits being determined. Two parallel planes with homogeneous surface reflectance are used. The round foreground and the background are separated by a distance shorter than the range resolution and both need to be perpendicular to the aiming direction of the applied instrument.

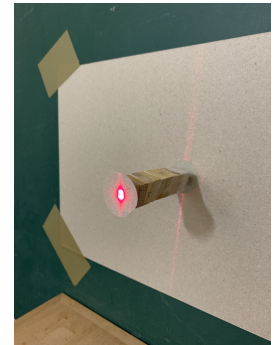

(a)

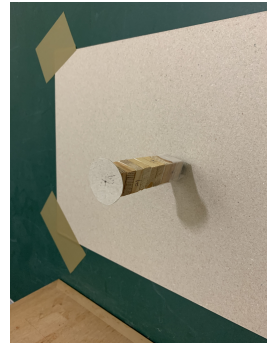

(b)
Figure 4. (a) The red area is the guidance of the laser beam, which appears larger than the foreground size (b) Experiment layout of case 1

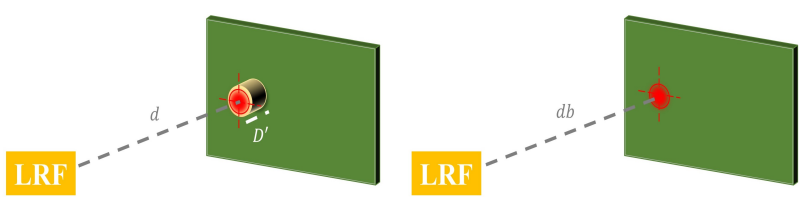

Figure 5. Illustration of case 1 experiment, where LRF stands for laser rangefinders

$$
r=d-\left(d b-D^{\prime}\right)
$$

$$
\begin{aligned}
& \text { where } \quad \begin{array}{l}
r=\text { ranging error } \\
d=\text { distance to the foreground } \\
d b=\text { distance to the background } \\
D^{\prime}=\text { depth of the foreground }
\end{array}
\end{aligned}
$$

As illustrated in Fig. 5, generally, $d$ should equal $d b-D$ '. However, when a foreground radius is smaller than a footprint radius, the measured distance will contain a ranging error $r$ resulting from mixed pixels effect. By comparing the $r$ with the rangefinder's precision, it is applicable to check whether the measurements under current foreground are affected by mixed pixels effect. Through changing the foreground radius, it 
verifies the footprint diameter corresponding to a specific object distance by identifying entangled ranging errors. After the footprint dimension is estimated, the divergence angle can then be calculated based on Eq. (2).

$$
\theta=\frac{f s}{d}
$$

where

$$
\begin{aligned}
& \theta=\text { divergence angle } \\
& f_{S}=\text { footprint diameter } \\
& d=\text { object distance }
\end{aligned}
$$

\subsection{Case 2: mixed pixels effect}

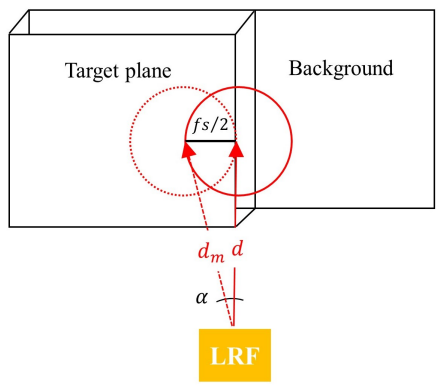

Figure 6. Illustration of decentering ranging method

Mixed pixels effect happens when a target point lies on or near discontinuous surfaces. This study proposes a decentering ranging method by shifting, toward avoiding mixed pixel effect, at least half of the divergence angle on the target plane. Yet, while eliminating the mixed pixels effect, an offset error and incidence angle effect are accompanied by such an operation. Fig. 7 demonstrates the ranging errors for different ranges (from 0 to $300 \mathrm{~m}$ ) when placing offset of half of divergence angles (from 0 to 0.5 degrees). Although the offset ranging errors can be corrected via Eq. (3), the ranging errors caused by incidence angle effect and axis offset have to be further considered, turning out that a joined correction of offset, axis offset, and incidence angle effect, as detailed in case 5 , would be more appropriate. The model of incidence angle effect is given in the next section.

$$
r_{\text {offset }}=d_{m}(1-\cos \alpha)
$$

where $\quad d_{m}=$ measured distance $\alpha=$ offset angle

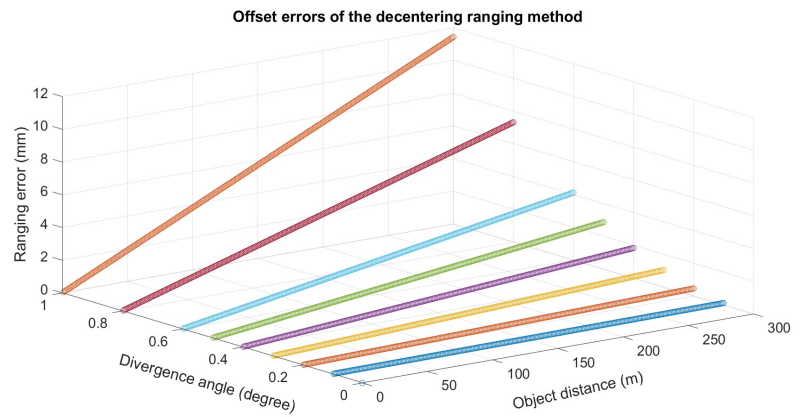

Figure 7. Offset errors corresponding to different divergence angles and object distances

\subsection{Case 3: incidence angle effect}

Fieldwork surveys may have measurements of target points in varied incidence angles and provoke ranging errors because of multi-range effect. However, without knowing the waveform or how the timing estimator is applied to the waveform of the ranging data, modeling and correcting the incidence angle effect cannot be obvious. Lichti et al. (2005) mentioned that the factors of the incidence angle effect ranging error include not only the incidence angles but also the object distances, and the two are in direct proportion. Analyzing how the footprints are shaped by the incidence angle $(\varphi)$ and ranging distance, as shown in Fig. 8(b), this study alternatively models the ranging errors of incidence angle effect as scaled $(s)$ ranging variation within the footprint by Eq. (4). Since divergence angle is a constant for each instrument, Eq. (4) can be further simplified as Eq. (5), which captured the very similar approach as given in (Baltsavias, 1999).

$$
\begin{gathered}
r_{\varphi}=s\left(\frac{1}{2} d_{m} \theta\right) \tan \varphi \\
r_{\varphi}=s_{\varphi} d_{m} \tan \varphi
\end{gathered}
$$

where $s_{\varphi}=\frac{1}{2} s \theta$

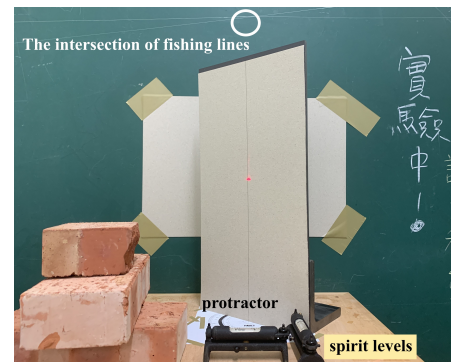

(a)

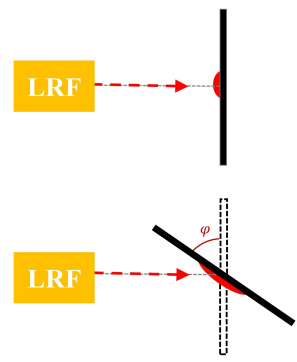

(b)
Figure 8. (a) Experimental layout of case 3; (b) Illustration of the larger incidence angle expanding the footprints

To collect ranging errors for modeling the incidence angle effect, a layout in support of both sufficient and precise observations is a must and it has been specifically designed in this study. As shown in Fig. 7, to fix a central axis used for rotating the target plane made of an acrylic board to designated angles and ensure the stability of the scene arrangement to be within the precision of applied instruments, a plane table, several pieces of bricks, and two spirit levels were employed. Besides, two fishing lines above the target plane intersect at the point very near the central axis to serve as a reference datum whenever the rotation of the target plane is tried. Among the collected observations, incidence angles are acquired by a protractor beneath the target plane. The ranging errors upon incidence angle effect are calculated taking the difference between the measured distance of targeted incidence angle and the one with zero incidence angle.

Furthermore, a footprint will expand with larger incidence angles and longer distances. Once the width of a target plane is shorter than the footprint diameter, the collected data will be distorted by mixed pixels effect. Therefore, before observations collection, the applicable distances and incidence angles should be evaluated by Eq. (6). After incidence angles, corresponding 
ranging errors and ranges are collected, adjustment based on Eq. (5) is performed and parameter $S_{\varphi}$ can be determined.

$$
f_{s}=\frac{d_{m} \theta}{\cos \varphi}
$$

To ensure the quality of the correction equation, the fitting results need to be checked by the following theoretical precision indicators (Kermarrec et al., 2018). The overall model quality can be statistically checked by the ratio of a posteriori variance factor to a priori variance factor. A residual vector shows the distribution of observations to a fitted function. And observations with outliers can be removed to refine the fitting quality. The standard deviations of model parameters provide how precise the parameters are estimated. It is crucial to check the uncertainties of the parameters with the instrument random errors to clarify whether the fitting results meet the quality requirements. Nevertheless, the above-mentioned indicators can only confirm the internal precision of the correction equation; therefore, external accuracy evaluation should be performed by reliable check data to verify whether the adjustment results are performed with sufficient quality. Root mean square error (RMSE) is employed to assess the true (or nearly true) error of the proposed model upon correction and helps reveal the systematic error, if existing, and thus remodel the correction function to refine the results. While root mean square difference (RMSD) is considered if the check data are with certain amount of errors.

\subsection{Case 4: incidence angle estimation}

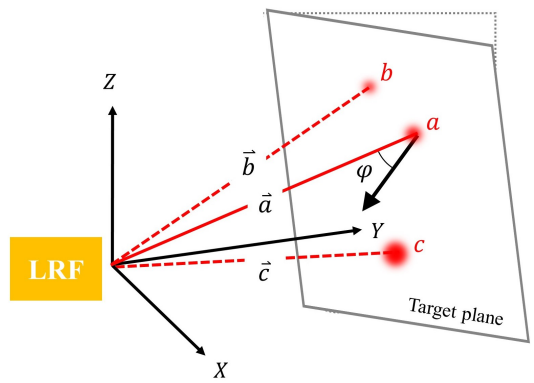

Figure 9. Illustration of incidence angle estimation approach

Practically, the incidence angle of ranging measurement is usually unknown. However, the relative geometry between a rangefinder and a target plane can be established by at least three non-collinear point measurements on the plane. As illustrated in Fig. 9, with the measurements of three points, including the target point $(a)$, three vectors basing on instrumental coordinate system can define the normal vector of the target plane and the incidence angle $(\varphi)$ of the target point can be calculated through Eq. (7). Yet, with incidence angle effect for each range measurement, an iterative computation algorithm as shown in Fig. 10 for correcting ranging errors of incidence angle effect while estimating the incidence angle was proposed.

$$
\varphi=\cos ^{-1}\left(\frac{\vec{a} \cdot[(\vec{b}-\vec{a}) \times(\vec{c}-\vec{a})]}{|\vec{a}||(\vec{b}-\vec{a}) \times(\vec{c}-\vec{a})|}\right)
$$

$$
\begin{aligned}
& \text { where } \left.\begin{array}{lll}
\vec{a} & \vec{b} & \vec{c}
\end{array}\right]=\left[\begin{array}{ccc}
x_{a} & x_{b} & x_{c} \\
y_{a} & y_{b} & y_{c} \\
z_{a} & z_{b} & z_{c}
\end{array}\right] \\
& =\left[\begin{array}{ccc}
d m_{a} \cos V_{a} \cos H_{a} & d m_{b} \cos V_{b} \cos H_{b} & d m_{c} \cos V_{c} \cos H_{c} \\
d m_{a} \cos V_{a} \sin H_{a} & d m_{b} \cos V_{b} \sin H_{b} & d m_{c} \cos V_{c} \sin H_{c} \\
d m_{a} \sin V_{a} & d m_{b} \sin V_{b} & d m_{c} \sin V_{c}
\end{array}\right]
\end{aligned}
$$

$\vec{a}, \vec{b}, \vec{c}=$ three vectors corresponding to three non-collinear points on the target plane $d_{m}=$ measured distance

$V=$ vertical angle

$H=$ azimuth angle

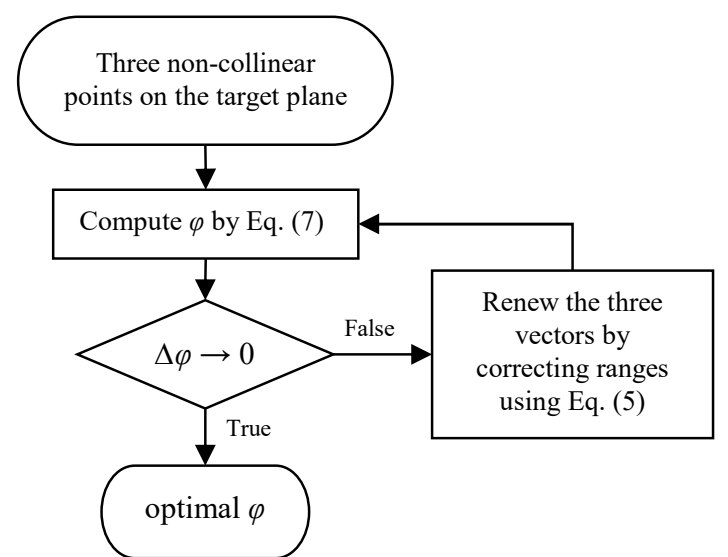

Figure 10. Iteratively updating incidence angle

\subsection{Case 5: offset correction}

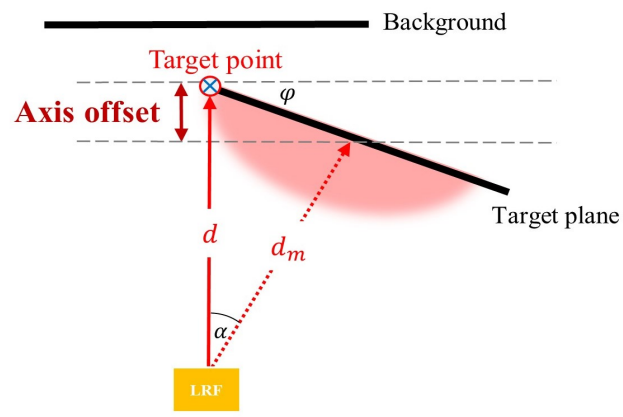

Figure 11. Illustration of axis offset

As shown in Fig. 11, when the incidence angle is known and the incidence angle effect has been modeled, the target point situated with mixed pixels effect can be corrected by Eq. (8) through an offset measurement (as mentioned in Section 2.2).

$$
d=d_{e}(\cos \alpha \pm \sin \alpha \tan \varphi)
$$

where $\quad d_{e}=$ corrected $d_{m}$ upon incidence angle effect

With the establishment of incidence angle estimation, correction of incidence angle effect, and placing offset measurement in need of tackling mixed pixels effect, Eq. (8) is indeed an integrated formula for correcting generalized mixed pixels effect. 


\section{EXPERIMENT RESULTS AND ANALYSIS}

To evaluate the effectiveness of the proposed workflow, two total stations equipped with the pulse time-of-flight laser ranging technique were employed. Tab. 1 displays the specification of the utilized equipment, and the experimental results are presented as follows.

\begin{tabular}{|c|c|c|}
\hline & $\begin{array}{c}\text { Trimble } \\
\text { M3 DR 2” }\end{array}$ & $\begin{array}{c}\text { Topcon } \\
\text { GPT-3002LN }\end{array}$ \\
\hline Illustration & $\pm 10 \mathrm{~mm}$ & $\pm 10 \mathrm{~mm}$ \\
\hline $\begin{array}{c}\text { Distance precision } \\
\text { from manufactures }\end{array}$ & $\pm 0.5 \mathrm{~mm}$ & $\pm 0.6 \mathrm{~mm}$ \\
\hline $\begin{array}{c}\text { Distance precision } \\
\text { estimated from } \\
\text { repeated observation }\end{array}$ & $0.2647^{\circ} \approx 16^{\prime}$ & $0.4647^{\circ} \approx 28^{\prime}$ \\
\hline $\begin{array}{c}\text { Divergence angle } \\
\text { estimated by case 1 }\end{array}$ & \\
\hline
\end{tabular}

Table 1. The specifications of the employed equipment

\subsection{Case 1: divergence angle estimation}

Fig. 12 depicts the collected data following case 1 experiment. At a distance of $6.924 \mathrm{~m}$, mixed pixels effect occurs with the ranging errors exceeding the precision of Trimble M3 DR 2" when the radius of foreground is shorter than $16 \mathrm{~mm}$ (Fig. 12(a)). Once the foreground radius exceeds $16 \mathrm{~mm}$, the ranging errors will be within the precision of Trimble M3 DR 2". Therefore, the divergence angle can be estimated using Eq. (2). Similarly, the divergence angle of Topcon GPT-3002LN (Tab. 1) is found by the same approach.

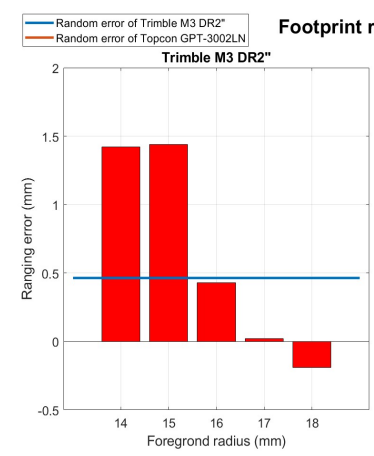

(a)

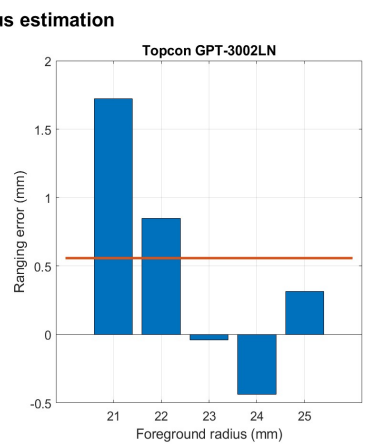

(b)
Figure 12. Data collected for estimating divergence angles. (a) Trimble M3 DR 2" with object distance $6.924 \mathrm{~m}$; (b) Topcon GPT-3002LN with object distance $5.670 \mathrm{~m}$

\subsection{Case 2: mixed pixels effect}

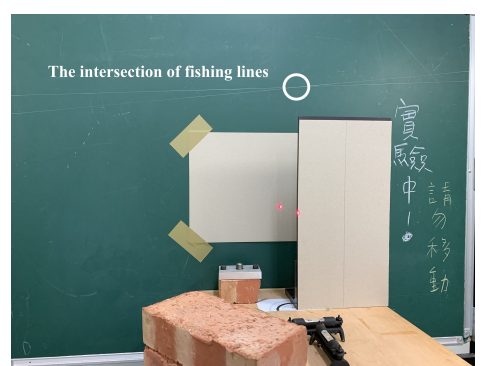

Figure 13. The scene layout of case 2
With the aid of a plane table, an acrylic board, two spirit levels, bricks and fishing lines, a strict layout can be set and the instrument was aimed to the target point located on the edge of the acrylic board (Fig. 13). According to Fig. 14, the results of case 2 reveal that after shifting half of the divergence angle acquired in case 1, the mixed pixels effect of the two instruments can be significantly eliminated, and both the remaining errors are within the random errors.

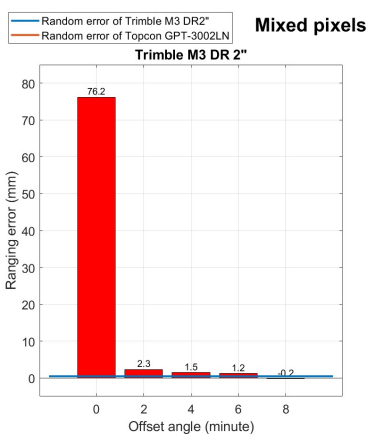

(a)

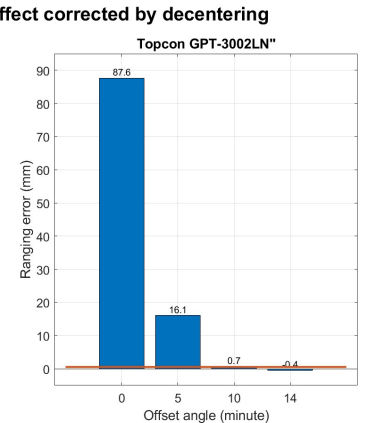

(b)
Figure 14. Mixed pixels effect correction. (a) Trimble M3 DR 2"; (b) Topcon GPT-3002LN

\subsection{Case 3: incidence angle effect}

To collect the ranging errors of case 3 under certain distances without being affected by the mixed pixels effect, the maximum corresponding incidence angle for a dimension-limited target plane needs to be calculated by Eq. (6). The width of a target plane used in this experiment is $200 \mathrm{~mm}$, and the workable conditions of the following experiments are presented in Fig. 15 and 16, where the red dash lines indicate the maximally allowable footprint diameter.

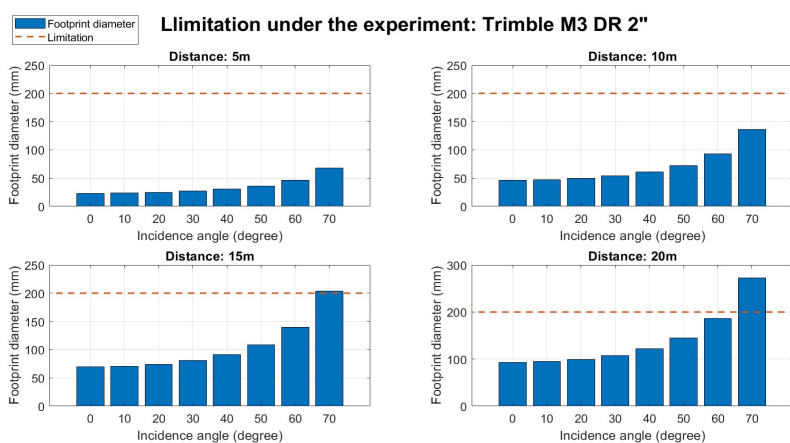

Figure 15. Limitation of acquiring case 3 ranging errors: Trimble M3 DR 2"

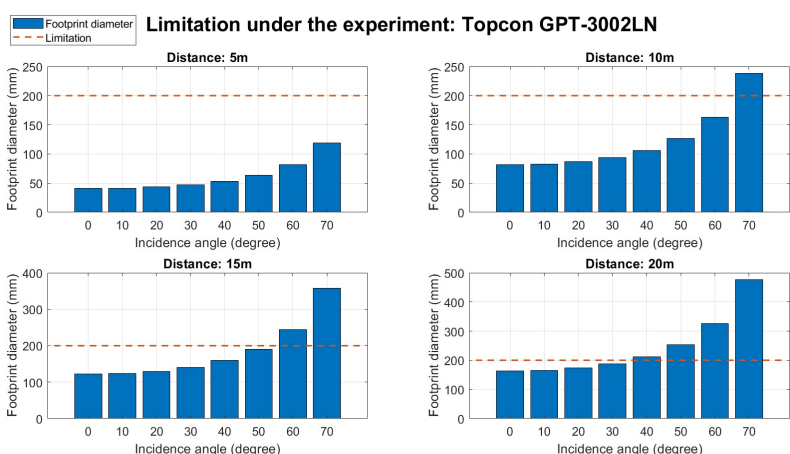

Figure 16. Limitation of acquiring case 3 ranging errors: Topcon GPT-3002LN 
With knowledge of the experimental limitation, the experiments were conducted under the scene layout of Fig. 8(a). By implementing the least-squares adjustment of indirect observations, the incidence angle effect can be modeled and the parameter can be estimated by Eq. (5). Total 34 data were collected and 12 of which were treated as check data to assess external accuracy. Tab. 2 shows the ranging error data of Trimble M3 DR 2" at specific incidence angles and distances, and the adjustment results.

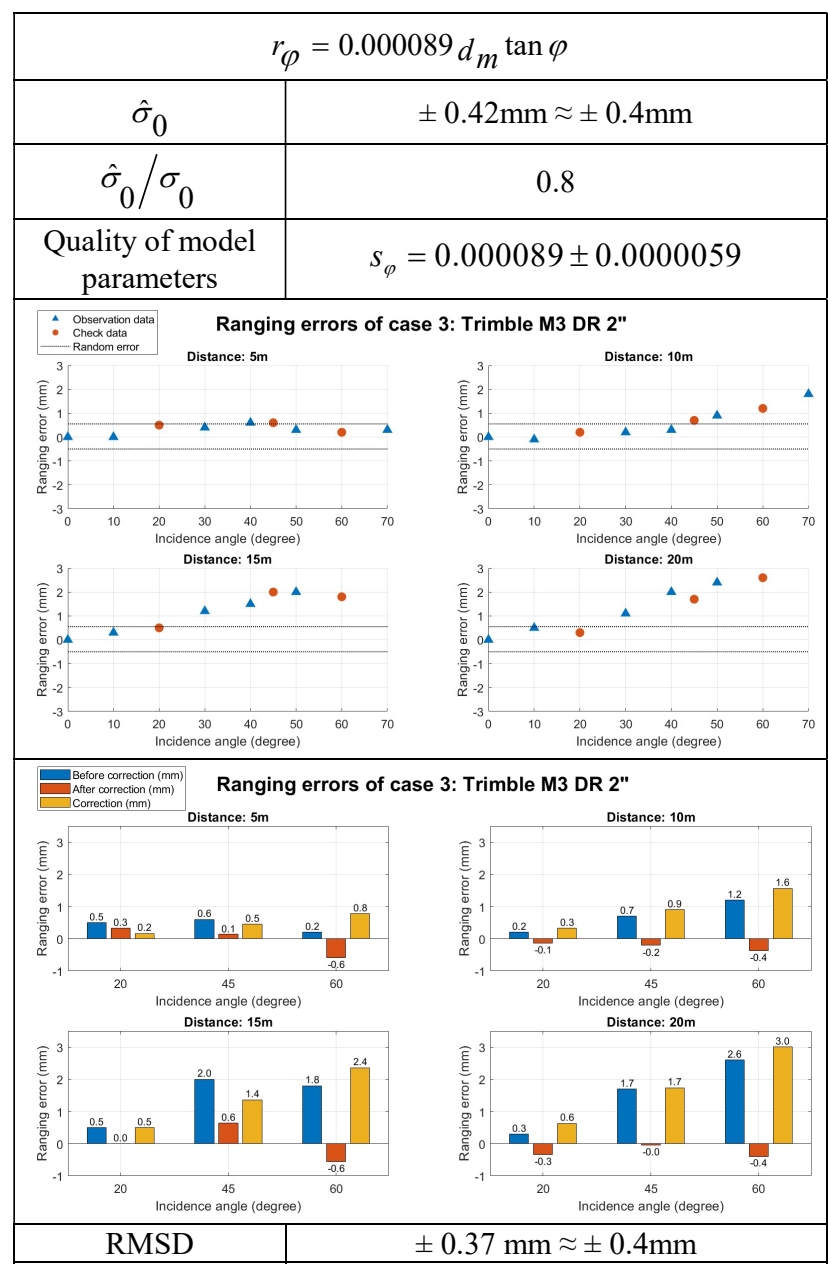

Table 2. Ranging correction results of case 3: Trimble M3 DR 2"

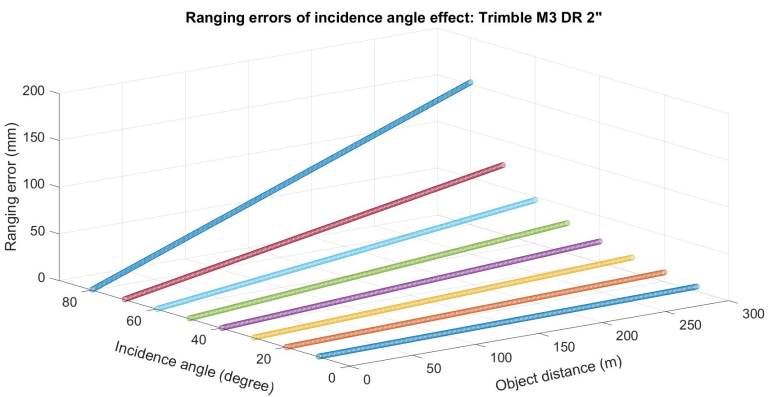

Figure 17. Ranging errors of case 3 under various incidence angles and distances: Trimble M3 DR 2"

Through estimating the parameter of Eq. (5), the correction equation of incidence angle effect is shaped. As shown in Tab. 2, after correcting the incidence angle effect, the RMSD drops to $\pm 0.4 \mathrm{~mm}$, showing the effectiveness of the model tackling the ranging errors caused by incidence angle effect. In addition, it indicates that the proposed method can improve the ranging results from mm-level to below mm-level even under a large incidence angle of observation. Fig. 17 offers a quite convenient visualization in realizing how incidence angles and ranging distances compose incidence angle effect of Trimble M3 DR 2".

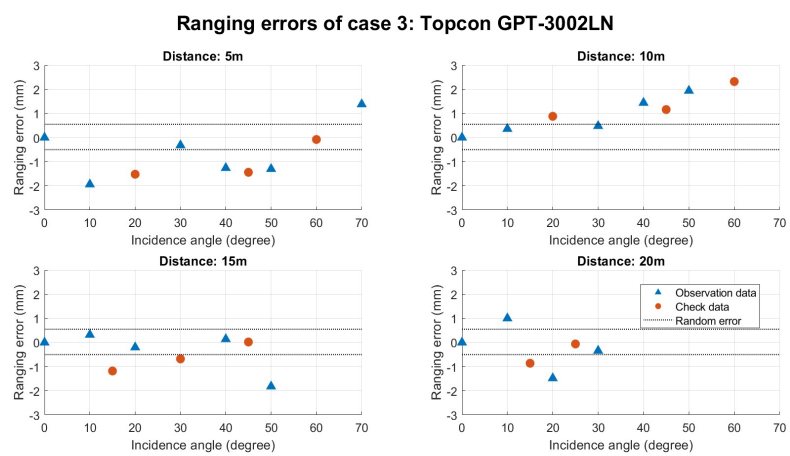

Figure 18. Incidence angle effect: Topcon GPT-3002LN

Fig. 18 reports the incidence angle effect of Topcon GPT3002LN. The ranging errors perform several slight bumps and no obvious trend can be drawn, i.e. with a larger incidence angle, ranging errors will increase or decline. The anomalous but insignificant ranging errors imply that the incidence angle effect of this instrument may not be reflected at short object distances. On the other hand, with high quality but insufficient data, the incidence angle effect of this instrument cannot be modeled yet.

\subsection{Case 4: incidence angle estimation}

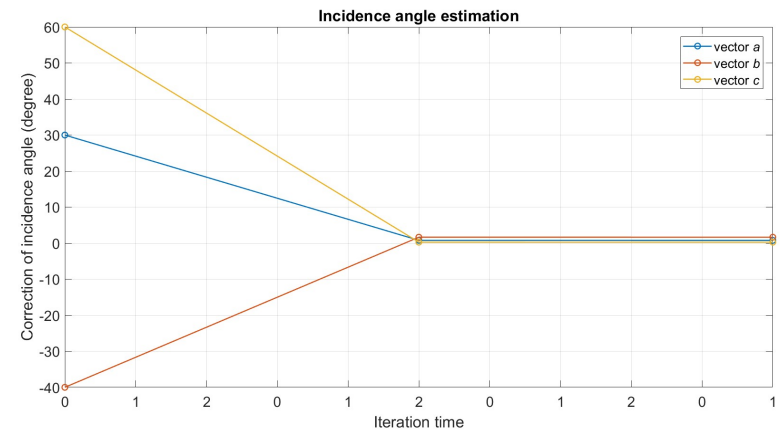

Figure 19. Result of iterative estimation of incidence angles: Trimble M3 DR 2"

Applying the proposed iterative estimation scheme to Trimble M3 DR 2", the incidence angle of a target point with about $5 \mathrm{~m}$ from the instrument reached convergent solution (Fig. 19).

\subsection{Case 5: offset correction}

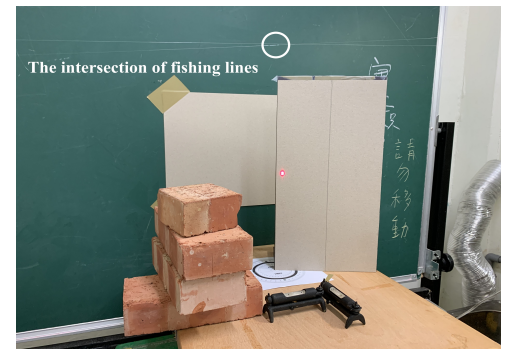

Figure 20. The scene layout of case 5, implementing the decentering ranging method 
After the data acquisition, as configured in Fig. 20, and offset correction, the comprehensive correction result of Trimble M3 DR 2" (Fig. 21) illustrates the validity and feasibility of the integrated model of generalized mixed pixels effect (Eq. (8)). Even though the remaining ranging errors (as evaluated by $\mathrm{RMSD}= \pm 1.5 \mathrm{~mm}$ ) still exceed the precision (with standard deviations below $1 \mathrm{~mm}$ ) estimated from repeated observation, they are already included in the precision provided by the manufactures (Tab. 1). In addition, comparing the ranging errors before and after the proposed correction model, it can be seen that this study performs a significant reduction of ranging errors under generalized mixed pixels effect.

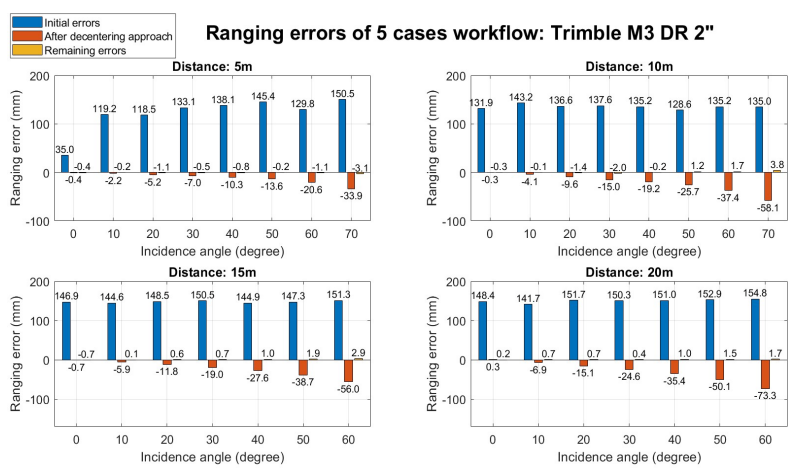

Figure 21. Integrated correction results: Trimble M3 DR2" $(\mathrm{RMSD}= \pm 1.5 \mathrm{~mm})$

Moreover, Fig. 22 describes the remaining errors without correcting the incidence angle effect (case 3). The overall RMSD of Fig. 22 is about 1 millimeter greater than the corrected version. It indicates that for optimizing the ranging results of Trimble M3 DR 2", the incidence angle effect must be taken into account.

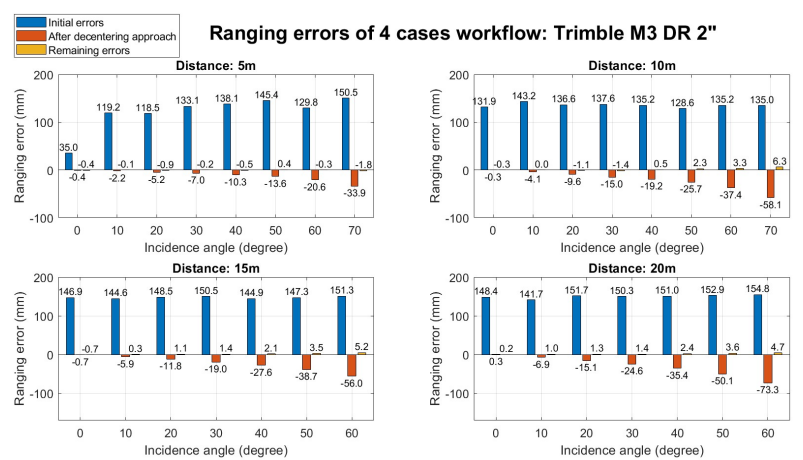

Figure 22. Integrated correction results without case 3 correction: Trimble M3 DR2" $(\mathrm{RMSD}= \pm 2.3 \mathrm{~mm})$

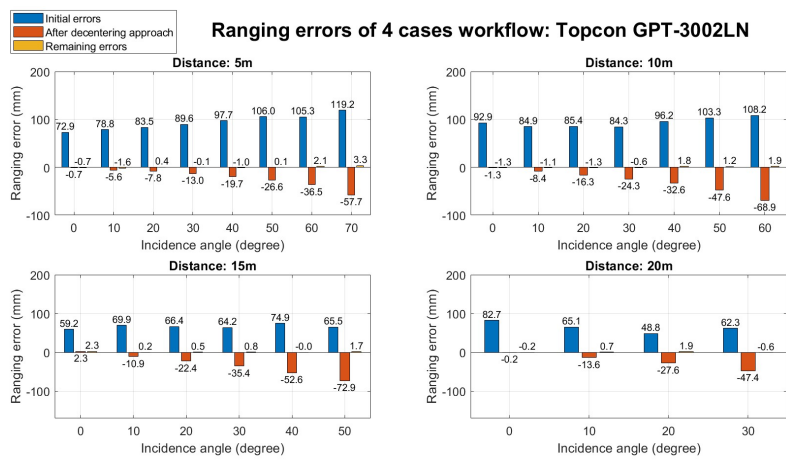

Figure 23. Integrated correction results without case 3 correction: Topcon GPT-3002LN (RMSD $= \pm 1.4 \mathrm{~mm})$
Since the incidence angle effect of Topcon GPT-3002LN has not yet fully estimated, a series of corrections have been made without considering incidence angle effect (Fig. 23). Even so, the RMSD of the remaining errors is only $\pm 1.4 \mathrm{~mm}$ under short object distances.

\section{CONCLUSION}

This study presented a laser ranging correction workflow, aiming to correct ranging errors under generalized mixed pixels effect without any physical waveform information. To restore the ranging quality, individual effects causing systematic errors are gradually analyzed by case studies. Two total stations with pulsed time-of-flight laser ranging technology were conducted to verify the effectiveness of the developed workflow. Based on the remaining errors of Trimble M3 DR 2", it shows that the proposed workflow significantly improves the ranging error by 97\%. Although the incidence angle effect of Topcon GPT$3002 \mathrm{LN}$ cannot be modeled yet due to insufficient data, a $60 \%$ improvement in ranging error has been still found.

\section{REFERENCES}

Abshire, J. B., McGarry, J. F., Pacini, L. K., Blair, J. B., Elman, G. C., 1994. Laser altimetry simulator. Version 3.0: User's guide.

Adams, M. D., 1993. Amplitude modulated optical range data analysis in mobile robotics. In: IEEE International Conference on Robotics and Automation, 8-13.

Adams, M. D., Probert, P. J., 1996. The interpretation of phase and intensity data from AMCW light detection sensors for reliable ranging. The International Journal of Robotics Research, 15(5), 441-458.

Baltsavias, E. P., 1999. Airborne laser scanning: basic relations and formulas. ISPRS Journal of Photogrammetry and Remote Sensing, 54(2-3), 199-214.

Hebert, M., Krotkov, E., 1992. 3D measurements from imaging laser radars: how good are they? Image and Vision Computing, 10(3), 170-178

Kaasalainen, S., Jaakkola, A., Kaasalainen, M., Krooks, A., Kukko, A., 2011. Analysis of incidence angle and distance effects on terrestrial laser scanner intensity: Search for correction methods. Remote Sensing, 3(10), 2207-2221.

Kermarrec, G., Alkhatib, H., Neumann, I., 2018. On the Sensitivity of the Parameters of the Intensity-Based Stochastic Model for Terrestrial Laser Scanner. Case Study: B-Spline Approximation. Sensors, 18(9), 2964.

Lichti, D. D., Gordon, S. J., Tipdecho, T., 2005. Error models and propagation in directly georeferenced terrestrial laser scanner networks. Journal of surveying engineering, 131(4), $135-142$.

Soudarissanane, S., Lindenbergh, R., Menenti, M., Teunissen, P J. G., 2009. Incidence angle influence on the quality of terrestrial laser scanning points. In Proceedings ISPRS Workshop Laserscanning 2009, 1-2 Sept 2009, Paris, France. 
Tan, K., Zhang, W., Shen, F., Cheng, X., 2018. Investigation of TLS intensity data and distance measurement errors from target specular reflections. Remote Sensing, 10(7), 1077.

Typiak, A., 2008. Use of laser rangefinder to detecting in surroundings of mobile robot the obstacles. In: Symposium on Automation and Robotics in Construction, 26-29.

Wolff, C., 2009: Radar tutorial (book 1): Radar basics. Western Pommerania: Achatweg3.

Xiang, J. C., Zhang, M. Y., 2001: Radar system. Publishing House of Electronics Industry. 